(REVIEW ARTICLE)

\title{
Most common coagulation disorders in recurrent pregnancy loss
}

\author{
Omer Ibrahim Abdallah Mohammed * \\ Deportment of Hematology, College of Medical Laboratory Sciences, Omdurman Islamic University, Khartoum, Sudan.
}

Publication history: Received on 11 June 2020; revised on 21 June 2020; accepted on 23 June 2020

Article DOI: https://doi.org/10.30574/wjarr.2020.6.3.0201

\begin{abstract}
Recurrent Pregnancy loss (RPL) represents a major health problem, with approximately 15-20\% of all clinically recognized pregnancies resulting in pregnancy unhappy end. Recurrent pregnancy loss (RPL) defined as 3 consecutive pregnancy losses before 20 weeks it affects approximately 1\% to $2 \%$ of women. Recurrent pregnancy loss exacts a devastating emotional toll on patients' lives. Each pregnancy loss brings with it a profound sense of loss and frustration. In this review we briefly address most common coagulation disorders in recurrent pregnancy loss.
\end{abstract}

Keywords: Recurrent Pregnancy loss; Blood Coagulation; Pregnancy

\section{Introduction}

Hemostasis represents a balance between pro- and anti-coagulant processes, with variations in this balance determining the net outcome. Significant physiological changes during pregnancy result in a hypercoagulable and hypofibrinolytic state that serves to protect the mother from bleeding complications at the time of placental separation [1]. Pregnancy is a period of reproduction during which a woman carries one or more live offspring from implantation of a fertilized zygote in the uterus throughout gestation. There are several physiological changes that occur in pregnancy. Physiology of a normal pregnancy involves major changes in both the coagulation system and hematological parameters. These changes appear to be related to the development of the uteroplacental circulation and provide a protective mechanism during delivery [2]. Recurrent pregnancy loss (RPL) is defined as two or more consecutive pregnancy losses before twenty weeks of gestation, which affects $13 \%$ of couples [3]. Women with inherited bleeding disorders face hemostatic challenges during various stages of pregnancy. Women who are carriers may have abnormally low factor levels and be at risk as well. Bleeding may occur at the time of delivery and postpartum, but the patient may also be at risk following spontaneous pregnancy loss, during diagnostic procedures, and during termination of pregnancy $[4,5]$. The function of the coagulation pathway is to keep hemostasis, which is the blockage of a bleeding or hemorrhage. Primary hemostasis is an aggregation of platelets forming a plug at the damaged site of exposed endothelial cells. Secondary hemostasis includes the two main coagulation pathways, intrinsic and extrinsic, that meet up at a point to form the common pathway. The common pathway ultimately activates fibrinogen into fibrin [6]. The most common inherited blood coagulation disorders are deficiencies of antithrombin III, protein C and protein S, Factor V Leiden mutation, and prothrombin gene mutation (G20210A).

\section{Prothrombin gene mutation (G20210 mutation)}

Prothrombin gene (G20210A) mutation is associated with an increased risk of thrombosis and it is the most identifiable risk factor for venous thrombosis and is in fact the second most common genetic defect for inherited thrombosis, with Factor V Leiden being the most common. It is an autosomal dominant disorder, with Heterozygotes being at a 3- to 11fold greater risk for thrombosis in both men and women and for all age groups. Although homozygosity is rare, inheritance of two 20210A alleles would increase the risk for developing thrombosis [7,8]. This mutation occurs as a result of the $\mathrm{G}$ to A transition at nucleotide 20210 in the prothrombin gene. Higher incidence of inherited thrombophilia

\footnotetext{
${ }^{*}$ Corresponding author: Omer Ibrahim Abdallah Mohammed
} 
factor prothrombin G20210A mutation has been the focus of some studies in women with RM and suggest that the prothrombin G20210A mutation, may be an unrecognized cause of RPL [9].

\section{Factor V Leiden G1691A mutation}

Factor V Leiden mutation is a result of an amino acid substitution of glutamine for Arginine at amino acid position 506 in the factor V molecule. During normal clotting activated protein C (APC) inactivates factor Va and VIIIa by cleavage at specific sites. In the presence of the mutation in factor $\mathrm{V}$, the cleavage of this factor is deprived, leading to enhanced thrombin generation and hence increased clot formation [10]. Women with this mutation are two to three times more likely to have multiple (recurrent) miscarriages or a pregnancy loss during the second or third trimester. Some research suggests that the factor V Leiden mutation may also increase the risk of other complications during pregnancy, including pregnancy-induced high blood pressure (preeclampsia), slow fetal growth, and early separation of the placenta from the uterine wall (placental abruption) .However, the association between the factor V Leiden mutation and these complications has not been confirmed. Most women with factor $V$ Leiden thrombophilia have normal pregnancies and Homozygotes are much less common but have a much higher thrombotic risk, around eight times increased risk [11]. The association between the FVL mutation and RPL seems stronger for non-recurrent second-trimester pregnancy loss compared with recurrent early pregnancy loss. Factor $\mathrm{V}$ Leiden mutation is the most common hypercoagulable disorder occurring in 5\% of the white population. This mutation leads to a form of factor $\mathrm{V}$ that when activated to factor Va is resistant to degradation by activated protein $\mathrm{C}$. There is increased procoagulants activity and therefore increased risk of Thromboembolism [12].

\section{Factor XII mutation}

Deficiency of coagulation factor XII is an autosomal recessive disorder whose clinical implications remain controversial. Factor XII is a serine protease that circulates in the plasma as an inactive zymogen and is involved in the initiation of the coagulation cascade. To date, few studies have analyzed coagulation factor XII activities in women with recurrent abortion. Some authors have found an association between severe factor XII deficiency and an increased risk for recurrent abortion, whereas other groups failed to show this association. Some studies find that, factor XII deficiency is strongly associated with primary recurrent abortion, and women with secondary recurrent abortion show a tendency toward factor XII deficiency [13].

\section{Methylenetetrahydrofolate Reductase C677T}

Methylenetetrahydrofolate reductase (MTHFR) is one of the main regulatory enzymes in the metabolism of homocysteine that catalyses the reduction of 5, 10-methylenetetrahydrofolate to 5-methyltetrahydrofolate. Mutations in MTHFR gene lead to decreased activity of enzyme and hyperhomocystinemia, which induces platelet aggregation through promotion of endothelial oxidative damage. Although there are several mutations within the MTHFR gene, but C677T and A1298C mutations are the two most common mutations, C677T transition is a missense mutation in the exons four of this gene, which converts an alanine to a valine codon (at codon 222) in the N-terminal catalytic domain of the protein leading to a thermo labile protein, with decreased enzymatic activity. Also, the C677T MTHFR polymorphism is responsible for a decreased MTHFR activity and associated with increased plasma homocysteine concentrations that are induced for a folate metabolism disturbance. The second mutation is MTHFR A1298C, which is associated with decreased activity of enzyme, but not with thermo ability. A1298C transversion is a point mutation in exon7, characterized by a glutamate to alanine substitution (at codon 429) within the C-terminal regulatory domain of the protein [14].

\section{Protein $\mathrm{C}$ and Protein $\mathrm{S}$ deficiencies}

The protein C (PC) pathway, with its cofactor protein S (PS), is an important natural antithrombotic mechanism. Both PC and PS deficiencies have been implicated in thrombophilia. The molecular basis for hereditary PC and PS deficiencies is highly heterogeneous, with a large spectrum of mutations that have various effects on the expression of the relevant allele. A small subset of patients who are homozygous or compound heterozygous for a PC gene mutation have severe thrombotic complications at birth, whereas onset occurs later in the other cases [15]. Many studies have tried to address this issue and there is still controversy regarding the importance of thrombophilia in fetal loss. Association is often difficult to uncover because of inherent study design issues in the pregnant population. Some previous study results conducted to show association of these factors and RMS reported that the different prevalence of these mutations and polymorphism in different populations depending on ethnic background could explain these differences [16]. 


\section{Antithrombin III deficiencies}

Antithrombin III (ATIII) is a nonvitamin K-dependent protease that inhibits coagulation by neutralizing the enzymatic activity of thrombin (factors IIa, IXa, Xa). Antithrombin III activity is markedly potentiated by heparin, the principal mechanism by which both heparin and low-molecular-weight heparin result in anticoagulation [17]. Antithrombin is a potent inhibitor of the reactions of the coagulation cascade. Although the name, antithrombin, implies that it works only on thrombin, it actually serves to inhibit virtually all of the coagulation enzymes to at least some extent. The primary enzymes it inhibits are factor Xa, factor IXa and thrombin (factor IIa). It also has inhibitory actions on factor XIIa, factor XIa and the complex of factor VIIa and tissue factor [18]. There are two primary types of antithrombin deficiency: type I and type II. Type I antithrombin deficiency is characterized by an inadequate amount of normal antithrombin present. In this case, there is simply not enough antithrombin present to inactivate the coagulation factors. In type II antithrombin deficiency, the amount of antithrombin present is normal, but it does not function properly and is thus unable to carry out its normal functions. In many cases, the antithrombin in type I deficiencies has a problem binding to heparin, although there have been multiple other changes to the antithrombin molecule described [19].

\section{Conclusion}

Most cases of RPL remain unexplained and inherited coagulation disorders causes account for majority causes among women [20]. There are many other causes can affect coagulation cascades during normal pregnancy like uncontrolled arterial hypertension (AHT), diabetic and infections, can compromises correct gestation development [21,22,23]. Inheritance blood coagulation factors has been shown to be a major cause of recurrent pregnancy loss, patients with RPL should be evaluated for clotting disorders, even in the absence of clinical signs because there were some studies concluded that many positive hemophilic causatives finding without any clinical signs

\section{Compliance with ethical standards}

\section{Disclosure of conflict of interest}

The author reports no conflicts of interest in this work.

\section{References}

[1] Millar C and Laffan M. (2012). Hemostatic changes in normal pregnancy. InDisorders of Thrombosis and Hemostasis in Pregnancy, 1-13.

[2] Mohamed AO, Hamza KM and Babker AM. (2016). Physiological changes in some hematological and coagulation profile among Sudanese healthy pregnant women. Int J Med Sci Public Health, 5(3), 525-8.

[3] Toth B, Jeschke U, Rogenhofer N, Scholz C, Würfel W, Thaler CJ, et al. (2010). Recurrent miscarriage: current concepts in diagnosis and treatment. J Reprod Immunol, 85, 2532.

[4] Gernsheimer TB. (2016). Congenital and acquired bleeding disorders in pregnancy. Hematology 2014, the American Society of Hematology Education Program Book, 232-235.

[5] Babker AM. (2020). The role of Inherited Blood Coagulation Disorders in Recurrent Miscarriage Syndrome. Journal of Critical Reviews, 7(1), 16-20.

[6] Chaudhry R and Babiker HM. (2018). Physiology, Coagulation Pathways. In StatPearls [Internet]. StatPearls Publishing.

[7] Khan S and Dickerman JD. (2006). Hereditary thrombophilia. Thrombosis journal, 4(1), 15.

[8] Babker AMAAA and Gameel FEMH. (2015). Molecular Characterization of Prothrombin G20210A gene Mutations In pregnant Sudanese women with spontaneous recurrent abortions. Rawal Medical Journal, 40(2), 207-209.

[9] Kardi MT, Yousefian E, Allahveisi A and Alaee S. (2018). Association of factor V Leiden and prothrombin G20210A polymorphisms in women with recurrent pregnancy loss in Isfahan Province, Iran. International journal of preventive medicine, 9.

[10] Cochran DL. (2000). Clinical Pathology Rounds: Factor V Leiden Mutation in Association With Deep Vein Thrombosis During Pregnancy. Laboratory Medicine, 31(4), 194-7. 
[11] Van Vlijmen EF, Veeger NJ, Middeldorp S, Hamulyák K, Prins MH, Büller HR and Meijer K. (2011). Thrombotic risk during oral contraceptive use and pregnancy in women with factor V Leiden or prothrombin mutation: a rational approach to contraception. Blood, 118(8), 2 12- Reddy RR, Mutreja D, Moorchung N, Mukhopadhyay I. Recurrent pregnancy loss: can factor V Leiden mutations be a cause. Obstetrics \& gynecology science, 62(3), 179-82.

[12] Pauer HU, Burfeind P, Köstering H, Emons G and Hinney B. (2003). Factor XII deficiency is strongly associated with primary recurrent abortions. Fertility and sterility, 80(3), 590-4.

[13] Babker AMAAA and Gameel FEMH. (2016). Methylenetetrahydrofolate reductase c677t polymorphism in Sudanese women with recurrent spontaneous abortions. Kuwait Medical Journal, 48(2), 100-104.

[14] Dixit A, Kannan M, Mahapatra M, Choudhry VP and Saxena R. (2006). Roles of protein C, protein S, and antithrombin III in acute leukemia. American journal of hematology, 81(3), 171-174.

[15] Battinelli EM, Marshall A and Connors JM. The role of thrombophilia in pregnancy. Thrombosis.

[16] Smith SA and Morrissey JH. (2008). Heparin is procoagulant in the absence of antithrombin. Thrombosis and haemostasis, 100(07), 160-162.

[17] Hyers TM. (2001). Antithrombotic therapy for venous thromboembolic disease. Chest, 119(1 Suppl), 176S-193S.

[18] Patnaik MM and Moll S. (2008). Inherited antithrombin deficiency: a review. Haemophilia, 14(6), 1229-39.

[19] Babker AMAAA, Elzaki SG and Dafallah SE. (2013). An observational study of causes of recurrent spontaneous abortion among Sudanese women. Int J Sci Res, 4, 1435-8.

[20] Eldour, Ahmed Abdalla Agab, et al. (2016). "Fibrinogen Levels in Hypertensive and Normotensive: A CrossSectional Study from El-Obied City, Sudan." Journal of Biosciences and Medicines, 4(2), 28-32.

[21] Babker AM and Gameel FE. (2014). The frequency of factor V leiden mutation among Sudanese pregnant women with recurrent miscarriage. Journal of American Science, 10(9).

[22] Fikri Benbrahim O, García Agudo R, Cazalla Cadenas F, Martínez Calero A and González-Spínola J. Diagnosis of secondary hypertension causing miscarriage during the first trimester of pregnancy. Nefrología (English Edition), 31(2), 229-31.

\section{How to cite this article}

Omer Ibrahim Abdallah Mohammed. (2020). Most common coagulation disorders in recurrent pregnancy loss. World Journal of Advanced Research and Reviews, 6(3), 229-232. 